\title{
La planificación de la actividad docente en el proceso de enseñanza-aprendizaje: Traducción y Derecho
}

\author{
Ana Gregorio Cano \\ Universidad de Granada \\ anagregcan@ugr.es \\ Daniel CASAs Agudo \\ Universidad de Granada \\ dcasas@ugr.es
}

\begin{abstract}
Resumen:
En este artículo pretendemos dar a conocer una propuesta práctica de una guía didáctica para el estudiante de Derecho Financiero y Tributario y para el de Traducción e Interpretación. En ella se desglosarán aspectos clave tanto desde el punto de vista del estudiante como del profesor, donde aparecerán todos los criterios necesarios y desglosados para poder aprovechar con el mayor éxito posible una asignatura. Asimismo, se abordarán los aspectos que garantizan las bases para un proceso de enseñanza-aprendizaje transparente, claro y de calidad.
\end{abstract}

Palabras clave: Guía didáctica; guía docente; Traducción; Derecho Financiero y Tributario; proceso de enseñanza-aprendizaje.

\section{Planning for teaching in the teaching-learning process: Translation and Law}

\begin{abstract}
:
This article shows a practical proposal for a study guide for Financial and Tax Law and Translation and Interpreting students. Some key aspects both from the student and the professor perspectives and the necessary criteria in order to achieve the greatest success will be explained and clarified. Furthermore, the basis for a transparent, clear and quality assessment will be highlighted.
\end{abstract}

Key words: Study guide; teaching guide; Translation; Financial and Tax Law; teaching and learning process.

Referencia normalizada:

Gregorio Cano, A. y Casas Agudo, D. (2014): La planificación de la actividad docente en el proceso de enseñanza-aprendizaje: Traducción y Derecho. Historia y Comunicación Social. Vol. 19. Núm. Especial Enero. Págs. 525-538.

Sumario: 1. Introducción. 2. La necesaria planificación de la actividad docente: la implementación de las guías didácticas en los nuevos planes de estudio. 3. Guía didáctica: análisis de un ejemplo práctico. 3.1. Las guías de trabajo autónomo. 3.2. Evaluación. 4. Conclusiones. 5. Referencias bibliográficas. 


\section{Introducción}

Las guías didácticas constituyen una fuente inicial de aproximación al conocimiento de la asignatura por parte del alumno, por lo que pueden ser entendidas como el inicio del diálogo de conocimientos que se entabla entre profesor y alumno. La inclusión de esta técnica como eje fundamental en muchas de las asignaturas se ha visto incrementada con la implantación de los nuevos grados como parte de la implementación del Espacio Europeo de Educación Superior (EEES). En dicho contexto, el sistema europeo de créditos implica una reformulación de la organización del currículo de la educación superior mediante su adaptación a los nuevos modelos de formación centrados en el estudiante.

Frente a la tradicional metodología docente de transmisión vertical (profesor-alumno) de los conocimientos a través de la clase magistral -conocimientos cerrados que, en hipótesis, le servirán para toda la vida- y su correlativa evaluación mediante el sistema de exámenes, las nuevas perspectivas metodológicas consideran que el aprendizaje debe considerarse como un continuum a lo largo de la vida (Lifelong Learning), de ahí que el nuevo paradigma docente sea "enseñar a aprender", por lo que fomentar las capacidades de auto-aprendizaje y el espíritu crítico de los alumnos se encuentren entre los nuevos objetivos educativos. Este cambio en la manera de concebir el modelo educativo debe llevar aparejada una correlativa evolución en la organización y en la metodología docente.

En el contexto de la implementación del crédito ECTS (European Credit Transfer and Accumulation System), la guía didáctica cobra una especial importancia ante el cambio de paradigma que conlleva en los nuevos planes de estudio. A través de ellas se podrán modular y programar las diversas actividades docentes, ajustándose a las directrices del plan. Las guías permitirán expresar cuáles son los conocimientos y competencias que tienen que alcanzar los alumnos, a través de qué vías y qué actividades deben desarrollar para tal fin.

En nuestro trabajo nos proponemos analizar dos guías didácticas: una diseñada en el seno del grado de Traducción e Interpretación y otra en el seno del Derecho, como ejemplo práctico para el futuro diseño de este tipo de guías en cualquier disciplina. Como veremos, dichas guías contienen la información necesaria tanto acerca del contenido como de la metodología, objetivos, actividades y criterios de evaluación de la asignatura, y son la base sobre la que se asienta gran parte del reto de la adaptación metodológica de la enseñanza universitaria al nuevo EEES y a los créditos ECTS.

\section{La necesaria planificación de la actividad docente: la implementación de las guías didácticas en los nuevos planes de estudio}

La idea de previsión, de anticipación, de planificación va asociada, invariablemente, a toda tarea responsable y trascendente. El concepto de planificación es consi- 
derado uno de los logros de la moderna tecnología educativa y se fundamenta en la convicción de que la educación, entendida como tarea racional y sistemática, exige conocer anticipadamente, de manera precisa, qué objetivos deben alcanzar los alumnos. Sólo en función de unos objetivos claros es posible planear una estrategia de aprendizaje (actividades) y unos procedimientos de control (evaluación).

La planificación cabe interpretarla en dos sentidos: a) como proceso (acción de planificar) y b) como producto (resultado de la planificación -guía didáctica o instrumento similar-). Por tanto cuando hablamos de planificación hacemos referencia al proceso que coordina objetivos, contenidos, medios y criterios de evaluación. Se ha de planificar la asignatura mediante instrumentos que orienten y faciliten el trabajo tanto de profesores como de los alumnos.

El sistema de planificación introduce importantes novedades respecto de los programas tradicionales. Estos últimos eran un índice de cuestiones ordenadas de un modo lógico (de contenidos, por tanto) que guiaba, fundamentalmente, la actividad del profesor. Dichos programas están asociados al modelo de profesor "impartidor" de contenidos. Frente a lo anterior, la planificación moderna se encuentra fundamentalmente orientada hacia los objetivos, guía el quehacer del alumno, es abierta y revisable y se asocia a un modelo de profesor "estimulador, facilitador y orientador" del aprendizaje.

Hoy día, los términos "planificación" y "diseño" sirven, por tanto, para designar un proceso de investigación, reflexión y análisis que permite tomar decisiones acerca de los objetivos a alcanzar para satisfacer unas determinadas necesidades de aprendizaje, los métodos, medios y tiempos necesarios para alcanzarlos y los procedimientos para evaluar el proceso de aprendizaje en su conjunto y sus resultados. El centro de atención de todo el proceso de diseño es el estudiante. Este desplazamiento del interés inicial por la enseñanza hacia el aprendizaje, que aparece como uno de los postulados del EEES, insistimos, obliga al profesorado a planificar y desarrollar la enseñanza de un modo diferente. No es suficiente con facilitar al alumno el material que tiene que estudiar sino que hay que indicarle cómo debe abordar el proceso de aprendizaje.

Se dice que un Plan didáctico es bueno cuando delimita con claridad los objetivos que pretende alcanzar; se adapta a los alumnos, conocidas sus características; expresa claramente las actividades a realizar; prevé el material o los recursos necesarios; incluye la evaluación de resultados; temporaliza las unidades y objetivos; permite que cada alumno camine a su propio ritmo; y considera la actividad del alumno como eje del proceso. Para conseguirlo, es preciso que cuando se afronta la ardua tarea de planificar una asignatura se tenga en cuenta los siguientes ideas orientadoras ${ }^{1}$ :

- ha de existir coherencia entre objetivos, medios y criterios de evaluación (racionalización);

- se ha de tener en cuenta, para cada fase del proceso de aprendizaje, los resultados anteriores (progreso escalonado); 
- la planificación debe traducirse en un documento abierto y revisable (flexibilidad);

- todos los que utilizan la planificación deben interpretar del mismo modo los datos y el alcance de las decisiones adoptadas (precisión unívoca);

- y los elementos de la programación deben adecuarse a las específicas condiciones: alumnos, material disponible, etc. (realismo).

Las actividades de planificación se traducen en la guía didáctica de la asignatura, que presenta la virtualidad de convertirse en un vehículo eficaz de comunicación entre el profesor y el alumno. Probablemente, el aspecto más trascendental es que el alumno conocerá de forma fehaciente la visión que el docente tiene acerca de la asignatura y de su modo de impartición. Y esto se podrá plasmar tanto a nivel general, como descendiendo al detalle de un tema concreto ${ }^{2}$. También constituye un vehículo importate en el que expresar los contenidos del autoaprendizaje que debe desarrollar el alumno. En concreto, permitirá una correcta planificación de las actividades no presenciales que podrá desarrollar el alumno. Estas guías didácticas se integran en lo que se ha denominado también "planificación operativa" (la que corresponde al profesor) para distinguirla de la planificación institucional que se concreta en las Guías ECTS.

Los contenidos de una guía didáctica son variados pero, en cualquier caso, deben recoger la información básica que precisa el estudiante para enfrentarse a la asignatura con garantías de éxito. En este sentido, además de incorporar una presentación de la misma y de sus objetivos, así como una delimitación de las competencias genéricas y específicas que los alumnos deben adquirir una vez culminado el proceso de aprendizaje, puede incluirse una mención a otros aspectos de utilidad e interés, tales como una breve información sobre el Departamento al que se adscribe la asignatura y del currículum del profesor encargado de impartirla; una referencia a aspectos organizativos de menor importancia, en principio, pero necesarios para el buen desarrollo de la asignatura (fichas, sistema de comunicaciones entre los alumnos y de éstos con el profesor a través de una de las plataformas y herramientas informáticas habilitadas al efecto por la Universidad, instrucciones para la elaboración de los trabajos en grupo, etc.); y otras referencias a cuestiones más relevantes, como son el propio contenido de la asignatura, programa de prácticas y cronograma del mismo, metodología, y criterios de evaluación.

La información anterior tiene que ser especialmente cuidada en lo que atañe al trabajo autónomo del estudiante puesto que uno de los cambios sustanciales impuestos por el modelo del EEES es el reconocimiento de un mayor protagonismo del alumno en su propia formación, menor dependencia, por tanto, del profesor y el uso de nuevas vías de relación, más allá del contacto "cara a cara" en el aula. En este nuevo contexto, el profesor tendrá que esmerarse en la confección de Guiones de Trabajo Autónomo y materiales de aprendizaje, que deben estar a disposición de los alumnos en forma impresa o digitalizada ${ }^{3}$. 
Debemos advertir que elaborar una guía de trabajo autónomo es una tarea laboriosa, que hay que realizar con esmero si se quiere alumbrar un documento que tenga verdadera utilidad. La guía tiene que ofrecer una secuencia de actividades trazada de tal modo que conduzca inexorablemente al aprendizaje. Estas actividades pueden ser muy variadas, desde reflexiones personales hasta ejercicios de aplicación o experiencias de otro tipo como visitas a instituciones, entrevistas, etc. Es un planeamiento cuidadoso y metódico del trabajo del alumno, con todas las referencias, fuentes y materiales necesarios para que aprenda por sí mismo. Algunos profesores solicitan posteriormente la evidencia del trabajo realizado en forma de una Carpeta de Aprendizaje (portfolio en la terminología anglosajona). Respecto a la estructuración de los contenidos, se ha de procurar tener en cuenta el nivel inicial del alumnado, la estructura de la materia manteniendo su coherencia interna; su relación con el curriculum general y el nivel final al que queremos que llegue el alumno. Se ha de tomar en consideración, además, las relaciones existentes con otras disciplinas para evitar posibles reiteraciones o repeticiones de contenido, así como para impedir que se produzcan omisiones, saltos, lagunas o incongruencias en el tratamiento de los mismos.

A un nivel distinto pero complementario al anterior, debemos destacar que la aprobación de los nuevos planes de estudio adaptados al EEES ha supuesto también, como no podía ser de otro modo, una revaloración de la programación académica, programación que puede encontrar también un cauce de expresión adecuada en las guías didácticas.

De entre las distintas directrices que hay que tener en cuenta a este respecto -en especial, las reativas a la organización de la docencia-, y por lo que se refiere en concreto a nuestra Universidad (la de Granada), en la "Guía para la elaboración de propuestas de planes de estudios de títulos oficales de grado" ", elaborada por el Vicerrectorado de Enseñanzas de Grado y Posgrado, se define el número de horas del crédito ECTS, que será de "25, que incluyen las enseñanzas teóricas, prácticas, así como las horas de trabajo individual -actividades académicas dirigidas-y de estudio del estudiante. Las horas lectivas presenciales se fijarán de acuerdo con las competencias establecidas, no pudiendo ser menos de un 20\% (5 horas) ni más de $40 \%$ (10horas)". En este entorno, la guía didáctica cobra singular importancia en los nuevos planes de estudio pues a través de ella se podrán modular y programar las diversas actividades docentes, ajustándose a las directrices del plan. Las guías permitirán de este modo expresar cuáles son los conocimientos y competencias que tienen que alcanzar los alumnos, a través de qué vías y qué actividades deben desarrollar a tal fin.

Aun siendo cierto que la elaboración de los nuevos planes de estudio ha podido no implicar necesariamente un impulso a la innovación docente (lo que depende especialmente del número de horas lectivas presenciales que se fijen dentro de las horquillas fijadas ${ }^{5}$ ), como señalan Mochón LóPEZ y RANCAÑo MARTín ${ }^{6}$, la guía seguiría siendo en todo caso un instrumento adecuado para reflejar la presencialidad o cuáles son las actividades no presenciales programadas, etc. Es decir, toda una información 
que permitirá al alumno afrontar la asignatura con una visión previa de ésta y de las estrategias pedagógicas escogidas por el docente.

\section{Guía didáctica: análisis de un ejemplo práctico}

De lo visto hasta ahora se deduce que tras el proceso de Bolonia y la implantación de los nuevos planes de estudio en España, el proceso de enseñanza-aprendizaje sufre un giro en cuanto a la concepción y el planteamiento de una asignatura en la mayoría de áreas de conocimiento, si bien es cierto que en algunas otras, como en el caso de Traducción e Interpretación Bolonia ha venido a afianzar un modo de trabajo en el aula que cuenta con una larga tradición en la disciplina. Sin embargo, el diseño de las guías didácticas - que no docentes- parece haber sido una cuenta pendiente tanto en el caso de las disciplinas que ya contaban con una dinámica como la que introduce Bolonia como para las que se sumergen en todos los cambios que acompañan a dicho proceso.

Existen diferentes estilos de aprendizaje por lo que la propuesta aquí presentada contará con diferentes enfoques, trabajos y métodos a la hora del diseño de una guía didáctica y máxime si tenemos en cuenta que nuestra propuesta pretende establecer unas bases generales que hagan posible el diseño de (casi) cualquier guía sin importar el área de conocimiento, de ahí que en el presente artículo estudiemos el caso específico del Derecho y también de la Traducción.

Por lo general, las guías didácticas tienen una estructura básica general, aplicable y extrapolable -con sus pertinentes adaptaciones- a casi cualquier asignatura o campo de conocimiento. En la siguiente tabla incluimos en el primer recuadro la propuesta de índice para una asignatura introductoria en el plan de estudios del Grado en Traducción, lo que supondría la primera toma de contacto de los estudiantes con la Traducción. En el segundo recuadro, el índice que aparece se corresponde con una asignatura obligatoria de tercer curso del grado en Derecho: 
Imagen 1. Ejemplo de los índices de las guías didácticas.

\begin{tabular}{|c|c|}
\hline \multicolumn{2}{|l|}{ fintise } \\
\hline Program genetaldo la atigntura & 3.7. \\
\hline 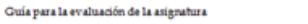 & 8.23 \\
\hline 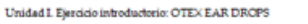 & 2622 \\
\hline 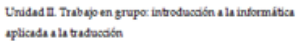 & 26 \\
\hline 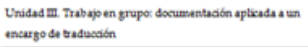 & $29 \cdot 3:$ \\
\hline 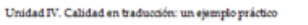 & 32,35 \\
\hline ENCARCOS1,2,3y4 & 36.37 \\
\hline 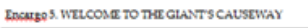 & $30+0$ \\
\hline 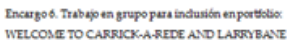 & $41+4$ \\
\hline Enargo 7. HAPPY BEN BL BLUE: & $45-4$ \\
\hline Encurgo 8. TVuCENCE & 43 \\
\hline Encargo9. POSmONDESCREMON1 & 40.50 \\
\hline Enargo 10. POSTMONDESCRIMDON2 & s.s3 \\
\hline 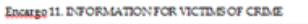 & \$4st \\
\hline 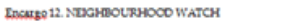 & $59-6$ \\
\hline
\end{tabular}

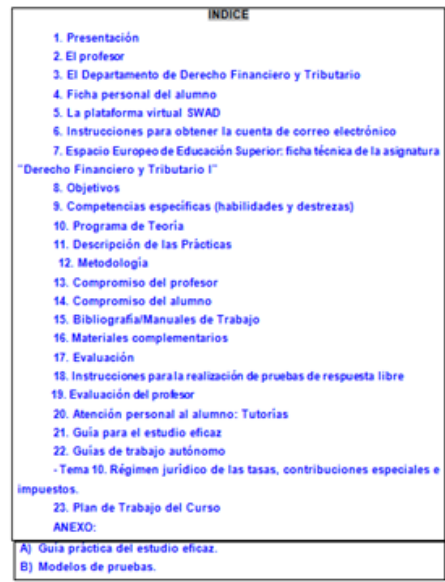

Como se desprende de la tabla y de la comparación entre un índice y otro, se puede apreciar que existen ciertas diferencias en cuanto a la estructura más allá de los contenidos, como es obvio. De este modo, en la guía didáctica de Traducción los apartados del 1 al 21 que en la propuesta de Derecho Financiero y Tributario se exponen de manera detallada, quedan recogidas en la propuesta de guía de Traducción en dos epígrafes, que son: programa general de la asignatura y guía para la evaluación.

De estas diferencias en cuanto a la inclusión de mayor o menor grado de detalle en los apartados que componen el índice de la guía se puede deducir que independientemente de cuántos epígrafes aparezcan, la espina dorsal es común y que se puede hacer más explícita o menos en función de las necesidades de cada asignatura, el estilo personal de cada docente, así como dependiendo del perfil de los estudiantes que compongan el aula.

En el presente artículo por cuestiones de espacio haremos un breve recorrido por algunos de los puntos básicos que a nuestro juicio ha de contener toda guía didáctica -como material de referencia para los estudiantes- y nos detendremos en dos aspectos esencialmente: las guías de trabajo autónomo y en la evaluación, que suele centrar la atención de los estudiantes antes incluso de conocer el nombre del profesor responsable de la asignatura, es decir, en la evaluación y cómo abordar este factor clave para el buen funcionamiento del proceso de enseñanza-aprendizaje.

Como en casi cualquier aspecto de la vida, para que algo llame la atención debe contar con una presencia atractiva, por este motivo, consideramos que el formato y en primer lugar la portada, por tratarse de la tarjeta de presentación de nuestra guía, debe transmitir cercanía, transparencia y adelantar que el contenido que encierra resulta reader-friendly. Cuando se confecciona cualquier guía didáctica no se puede perder de vista que el fin último, que nuestros destinatarios no son otros colegas de 
departamento o gremio, sino que diseñamos ese material para el óptimo desarrollo de la asignatura en cuestión y para que los estudiantes lean con atención y comprendan todo aquello que incluyamos en la guía.

Tras la elección de la portada, aparece el índice (véase imagen 1) en el que se recogen todos los apartados de nuestra guía. A continuación, se puede incluir una contextualización de la asignatura a la que se refiere la guía, resumir en términos generales para qué sirve, cuál es el fin de que se incluya en el plan de estudios y en el momento (semestre y curso) en el que se estudia en relación al resto de asignaturas, por ejemplo.

Asimismo, otro aspecto que puede resultar interesante desde el punto de vista de los estudiantes es un breve resumen del perfil del docente. En este apartado no se trata de incluir el currículum propiamente dicho, sino de que el profesor se dé a conocer un poco más a los alumnos, es decir, además de abordar aspectos académicos (perfil profesional, léase formación, experiencia docente, investigadora y de gestión, por ejemplo), sino ofrecer una visión más personal:

Soy Profesor en el Dpto. de Psicología Experimental (Universidad de Granada, UGR). Estudié la licenciatura de Psicología (1994-1998) en la UGR. Realicé los cursos de doctorado (1998-2003) y me doctoré en Psicología Experimental y Neurociencias del Comportamiento (2003, UGR) en torno a procesos psicolingüísticos $\mathrm{y}$ de memoria en personas bilingües y en traductores profesionales. Durante los años 2003-2005 realicé estancias de investigación postdoctoral en Estados Unidos sobre los procesos cognitivos y las bases neurofisiológicas asociadas al bilingüismo (University of Arizona y Penn State University). En el año 2005 regresé a la UGR, mediante una plaza de profesor ayudante doctor (años 2005-06), un contrato Ramón y Cajal (años 2006-2011) y una plaza de profesor titular (año 2011). Comencé las tareas docentes siendo profesor de prácticas durante los años 2000-2003 en el ámbito de la psicología del lenguaje y de la memoria. Desde el año 2005 he sido profesor de la asignatura Psicología del Lenguaje de manera continuada en varias titulaciones (magisterio -especialidad de audición y lenguaje-, psicología, logopedia). Además he sido docente en varios másteres de la UGR (traducción, neurociencia). Durante todos estos años, además de la vertiente docente, he realizado investigación sobre bilingüismo, traducción, control cognitivo, memoria, cognición numérica (y un largo etc.); mediante la dirección de tres proyectos del plan nacional de investigación. Además de las tareas docentes e investigadoras, otras actividades me gratifican día a día. Soy un enamorado de la música. Estoy abierto a cualquier género musical pero confieso mi debilidad por el rigor matemático del barroco alemán (J. S. Bach). Por otro lado, me considero un aprendiz (bastante autodidacta) de la guitarra española (algunos pinitos, aciertos y bastantes notas desafinadas salen de mi guitarra), y un neófito de la agricultura urbana: este verano he recolectado mis primeros higos chumbos (todo un reto en una terraza de 30 metros cuadrados en un $6^{\circ}$ piso). Esta pequeña presentación me gustaría resumirla en una frase: Soy profesor de algunas cosas de la vida y aprendiz de la mayoría. (Camacho, 2013: 11)

En cuanto a los apartados relativos a objetivos de la asignatura, competencias o temario se pueden extraer de las guías docentes que son al fin y al cabo el punto de referencia a la hora de diseñar una guía didáctica en concreto. No todo lo que se encuentra en la guía didáctica está presente en la guía docente, pero sí al revés, ya 
que no se puede olvidar que la guía didáctica pretende ser un documento de ayuda y referencia para el estudiante desde una perspectiva menos burocrática y en un tono menos formal que el que se utiliza en las guías docentes disponibles.

A continuación, procedemos a explicar los dos puntos fuertes del presente artículo, a saber: las guías de trabajo autónomo y la evaluación.

\subsection{Las guías de trabajo autónomo}

Las guías de trabajo autónomo son herramientas que permiten como su propio nombre indica la autonomía en el proceso de enseñanza-aprendizaje. Se trata de que el estudiante desarrolle su ritmo y método a la hora de adquirir y desarrollar el conocimiento en el seno de una determinada asignatura, al incluir esta herramienta como parte vertebradora de una asignatura se apuesta por el aprendizaje por descubrimiento del estudiante, ya que dichas guías se articulan en torno a técnicas de trabajo intelectual y de investigación, en ellas tienen cabida tareas tanto individuales como grupales, experiencias curriculares y extracurriculares, por lo que el interés desde el punto de vista del aprendizaje es incalculable.

La guía de trabajo autónomo se caracteriza por los siguientes aspectos, a saber (adaptado de RoMERO y CRISOL, 2012):

- Es un instrumento que el profesor pone en manos del estudiante para orientarle en sus tareas de descubrimiento y aprendizaje.

- Se trata de una relación de actividades que el estudiante debe desarrollar en su afán por buscar conocimientos, resolver problemas o adquirir nuevas destrezas.

- No son fichas, listas o relaciones de cuestiones, actividades o tareas repetitivas para casa o para clase que se resuelven copiando de un material previamente designado.

- Es un compromiso de trabajo para una unidad de tiempo debidamente acotada y del que el estudiante se responsabiliza de forma independiente.

- Implica una cuidadosa selección de sugerencias para motivar el aprendizaje y suscitar creatividad, iniciativa, actitudes de investigación, espíritu crítico, desarrollo de habilidades y adquisición de conocimientos.

La estructura que proponemos para la elaboración de una guía de trabajo autónomo puede contener los apartados que se desarrollan en la siguiente imagen, a saber: tema y contenidos, objetivos, actividades y bibliografía. El tema hace referencia al bloque al que pertenece dentro de la asignatura, así como los contenidos que conforman dicho tea; los objetivos que se pretenden alcanzar por medio de dicha guía de trabajo autónomo; las actividades que conforman el grueso de cualquier guía de este tipo, donde se recogen una secuencia de actividades obligatorias y voluntarias, y; por último, la bibliografía donde se recomienda facilitar una serie de obras de consulta de relevancia para poder llevar a cabo las actividades propuestas. 
Imagen 2. Ejemplo de una guía de trabajo autónomo.
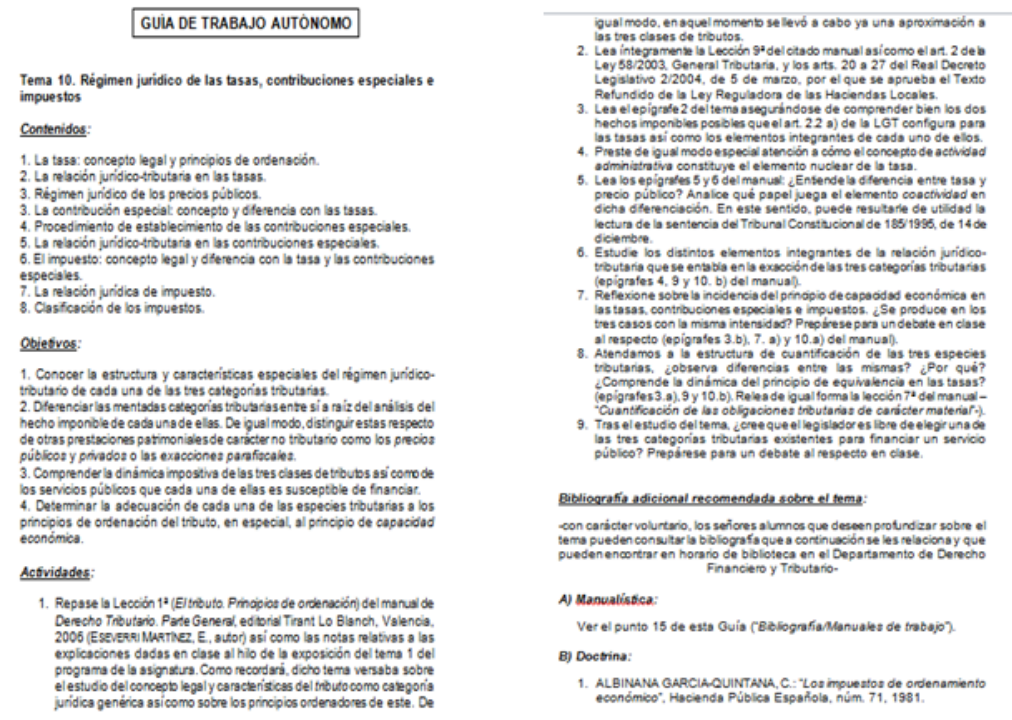

\subsection{Evaluación}

La evaluación suele ser uno de los aspectos que más preocupa a los estudiantes cuando tienen su primer contacto con una nueva asignatura. Para que no resulte una causa de estrés ni de preocupación, consideramos clave incluir en la guía didáctica toda la información referente a la evaluación y su dinámica para la asignatura correspondiente. Existen diferentes estilos de aprendizaje por lo que cabe justificar la elección de los diferentes enfoques y métodos a la hora de evaluar. De todo lo anterior se deduce que resulta de vital importancia que el docente diseñe de manera reflexionada y detenida tanto los materiales como los diferentes trabajos, encargos y exámenes que aportarán los datos necesarios para valorar la adquisición de conocimiento para mostrar el aprovechamiento de lo estudiado en la asignatura. Asimismo, es conveniente entender la evaluación - por parte de los estudiantes- como parte del proceso de adquisición de conocimiento, no como el fin de la asignatura.

La evaluación hace posible que el estudiante obtenga feedback del conocimiento, de sus puntos débiles y/o fuertes, y ayuda a que ejecute convenientemente los objetivos de la asignatura. Por otro lado, y a veces lo más importante para los estudiantes, la evaluación aporta una nota numérica del/al estudiante. Por último, y en muchas ocasiones un aspecto que no se aborda y se olvida, existen algunos tipos de evaluación que proporcionan información vital para el profesor mediante una evaluación realizada por el estudiante del proceso de enseñanza. 
La evaluación puede ser de varios tipos: diagnóstica, formativa y/o sumativa. A continuación se ofrece una breve definición de cada una de ellas:

- La evaluación diagnóstica es aquella que proporciona información al profesor sobre la aptitud y el nivel inicial de los estudiantes de cara a la asignatura en cuestión y posibilita la identificación de (posibles) problemas de aprendizaje.

- La evaluación formativa proporciona al alumno retroalimentación durante el proceso de aprendizaje y el desarrollo del mismo.

- La evaluación sumativa proporciona una nota alcanzada en cuanto al cumplimiento del estudiante en lo que respecta a los resultados de aprendizaje pretendidos por la asignatura.

En este artículo no ofrecemos un modelo de evaluación por considerar que la complejidad y amplitud del tema requeriría dedicar una publicación expresamente a la evaluación, aunque sí apostamos por la combinación de varios tipos de evaluación. A continuación incluimos un recuadro que hemos comprobado goza de una buena acogida por parte de los estudiantes en lo que se refiere a la evaluación, ya que se aborda la evaluación y el día del examen de una manera directa y clara:

Imagen 3. Evaluación: consejos prácticos de cara al día del examen.

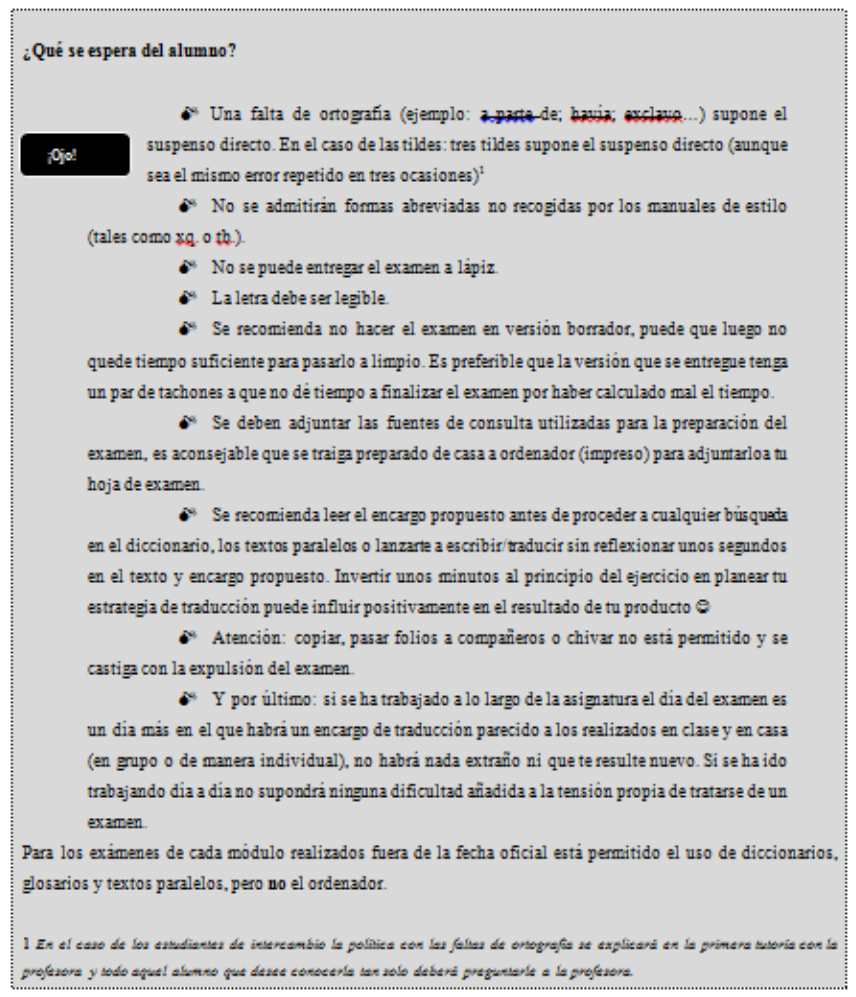




\section{Conclusiones}

De acuerdo con las nuevas directrices marcadas por el proceso de convergencia europea, resulta necesario que se lleve a cabo por parte del profesor una cuidadosa planificación de la enseñanza universitaria (idea de planificación o de diseño que se asocia con una docencia de calidad), que integre las variables relevantes del proceso de enseñanza-aprendizaje y que suministre a los alumnos la información necesaria acerca de lo que se trata de conseguir, cómo lograrlo y de qué modo se van a verificar los resultados, acrecentando con ello, en la medida de lo posible, su motivación, y reforzando al mismo tiempo el papel estimulador, facilitador y orientador del aprendizaje que corresponde al profesor.

La mentada planificación, que también se asocia con la idea de una docencia de calidad, debe estar inspirada, según el EEES, en la idea de que lo verdaderamente importante es lo que el alumno aprende, más que el tiempo que dedica a ese aprendizaje. Asimismo debe estar basada en la delimitación precisa de las competencias que el alumno deberá adquirir una vez culminado el proceso de aprendizaje, lo que, a su vez, exige relacionar armónicamente la teoría con la práctica tanto académica como profesional. Es necesario, pues, que los profesores universitarios posean los conocimientos y herramientas necesarios para abordar la tarea de planificación con garantías de éxito y que ésta se traduzca en la guía didáctica de la asignatura, de acuerdo con las finalidades de la convergencia europea.

\section{Bibliografía}

CAMACHO PÉREZ, S. "Planificación didáctica. Documento de trabajo para profesores universitarios", documento disponible en el siguiente enlace web: http:// calidad.ugr.es/pages/secretariados/form_apoyo_calidad/programa_iniciacion docencia/iniciacion3index [consultado el 1-10-2013].

(2013). Material inédito del curso "Planificación de la Docencia Universitaria por Competencias y Elaboración de Guías Didácticas" (5 $5^{\mathrm{a}}$ Edición) Guía Didáctica. Vicerrectorado para la Garantía de la Calidad Secretariado de Formación y Apoyo a la Docencia: Universidad de Granada.

CASAS AGUDO, D. (2010) Guía didáctica de la asignatura Derecho Financiero y Tributario I. Grupo G. Curso 2009/10.

GREGORIO CANO, A. (sin publicar) Material inédito. Guía didáctica de la asignatura Traducción B-A inglés. Curso 2012/13.

MOCHÓN LÓPEZ, L. y RANCAÑO MARTÍN, Ma . A. (2009). La enseñanza del Derecho en el Espacio Europeo de Educación Superior: dos ejemplos de guías didácticas, Grupo Editorial Universitario.

ROMERO LÓPEZ, Ma . A. y CRISOL MOYA, E. (2012). "Las guías de aprendizaje autónomo como herramienta didáctica de apoyo a la docencia". En: Escuela Abierta, $\mathrm{n}^{\circ}$. 15, 9-31. 


\section{Notas}

1 CAMACHO PÉREZ, S. "Planificación didáctica. Documento de trabajo para profesores universitarios", documento disponible en el siguiente enlace web: http://calidad.ugr.es/pages/secretariados/ form_apoyo_calidad/programa_iniciacion_docencia/iniciacion3index [consultado el 1-10-2013].

2 MOCHÓN LÓPEZ, L. y RANCAÑO MARTÍN, Mª A. (2009). La enseñanza del Derecho en el Espacio Europeo de Educación Superior: dos ejemplos de guías didácticas, Grupo Editorial Universitario, pág. 32.

3 El trabajo autónomo constituye una alternativa eficaz a los modelos directivos clásicos o modernos, suscitadores de aprendizajes receptivos. Basado en las aportaciones del aprendizaje por descubrimiento, subraya la intervención del alumno en el proceso de búsqueda y hallazgo de respuestas personales a problemas de contenido de muy variado carácter. Además, posibilita la autorregulación del aprendizaje, desarrolla en el alumno la responsabilidad por su propia formación y genera motivación intrínseca asociada a la satisfacción por la realización de la tarea.

En el aprendizaje por descubrimiento, el contenido esencial de lo que debe ser aprendido no se facilita en su forma final sino que tiene que ser descubierto por el sujeto. Los rasgos que definen el aprendizaje por descubrimiento son: acción fundamental del alumno, mínima intervención del profesor y producción de algo que no está en los datos.

El alumno trabaja sin una dependencia directa del profesor, investiga, analiza datos, consulta fuentes, organiza materiales, sintetiza ideas, elabora informes, presenta oralmente resultados, etc. Esto significa que su puesta en práctica requiere una tarea previa de instrumentación: el estudiante tiene que estar en posesión de una serie de técnicas que le permitan trabajar independientemente y conocer la nueva metodología.

4 Documento disponible en: http://vicengp.ugr.es/pages/normativa/guiaparalaelaboraciondepropuestasdeplanesdeestudiosdetitulosoficialesdegrado. [Consultado el 1-10-2013].

5 Piénsese en el caso en el que la presencialidad sea establecida en el máximo (10 horas por crédito ECTS), en ese caso se estaría adoptando una decisión que no favorecería la renovación pedagógica pues la presencialidad se identificará con las clase magistral, que implicará, por lo general, que el alumno tenga que dedicar una parte importante de su tiempo al estudio de la materia explicada. Así ues, en ese supuesto, el tiempo del crédito ECTS quedaría empleado en gran medida en las actividades docentes clásicas (clase magistral más estudio del alumno).

6 MOCHÓN LÓPEZ, L. y RANCAÑO MARTÍN, Mª A. (2009). Op. cit., p. 32.

\section{Los autores}

Ana Gregorio Cano. Profesora de Traducción e Interpretación. Universidad de Granada. Departamento de Traducción e Interpretación. Calle Buensuceso, 11. Granada, C.P. 18002. Traductora e Intérprete Jurado de Inglés. Becaria de personal docente e investigador predoctoral de la Junta de Andalucía en el Departamento de Traducción e Interpretación de la Universidad de Granada. 03/2008: Suficiencia Investigadora (DEA R.D. 778/1998). Tesis doctoral en Didáctica de la Traducción en curso. 
Daniel Casas Agudo es Doctor Europeo en Derecho por la Universidad de Bolonia (2006) y Profesor Investigador del Programa de Incorporación de Doctores de la Universidad de Granada en el Departamento de Derecho Financiero y Tributario de la misma Universidad. Está acreditado a Profesor Titular de Universidad (ANECA). 